Sharif University of Technology
Scientia Iranica
SCIENTIA

\title{
Evaluation of planing craft maneuverability using mathematical modeling under the action of the rudder
}

\author{
S. Hajizadeh, M.S. Seif* and H. Mehdigholi \\ School of Mechanical Engineering, Sharif University of Technology, Center of Excellence in Hydrodynamics and Dynamics of Marine \\ Vehicles, Tehran, Iran.
}

Received 28 August 2015; received in revised form 10 November 2015; accepted 25 April 2016

\section{KEYWORDS \\ Maneuvering; \\ Modeling; \\ Planing craft; \\ Rudder.}

\begin{abstract}
In the recent years, different mathematical models have been suggested for maneuvering of displacement vessels, which are capable to estimate maneuvering of the vessel with acceptable precision. But, simulation of planing craft maneuverability through a mathematical model has not been developed yet. In this paper, a mathematical model is developed for maneuvering of the planing craft by including the rudder forces and moments. Different maneuvers, such as straight-line stability, course keeping, and turning circle, are executed through the mathematical model. Simulation results are validated with the published experimental results and it is shown that they are in good agreement. Finally, the influence of rudder angle on maneuverability of planing craft is studied and, also, the effect of aspect ratio has been investigated. The mathematical model and hydrodynamic coefficients presented in this paper can be applied for the optimization of planing craft maneuvering and the course control purposes.
\end{abstract}

(C) 2017 Sharif University of Technology. All rights reserved.

\section{Introduction}

The hydrodynamic phenomenon involved in the maneuver of a planing craft causes difficulties in the simulation of its maneuver using the mathematical models. Several parameters affecting the maneuvering of the planing crafts are yet to be known and cannot be mathematically modeled. In high speeds, coupling of the motions of the vessels in horizontal, vertical, and transverse planes is significant and cannot be ignored.

The first maneuver modeling goes back to Davidsons [1]. He derived the maneuver equations and showed the complicated relation between the turning ability and path keeping in maneuver. Two main theories have been proposed for maneuver modeling, which

\footnotetext{
*. Corresponding author. Tel.: +982166165549;

Fax: +982166165563

E-mail addresses: Hajizadeh@mech.sharif.edu (S.

Hajizadeh); seif@sharif.edu (M.S. Seif); mehdi@sharif.edu

(H. Mehdigholi)
}

are still applicable and popular, namely, Abkowitz and MMG (Maneuvering Models Group, Japan) [2]. Abkowitz theory includes prediction of forces exerted on the vessel as a function of vessel's characteristic motion and integration of motion equation to find the real path of a maneuvering vessel.

With the advances in digital computing, simulations became a great substitution of model testing and significant changes in application of control theories to maneuvering occurred [3].

In the recent years, as new high-speed vessels entered the market, there have been numerous numerical and experimental studies regarding maneuvering of the vessel. The first experiment was performed on a self-propelled semi-displacement vessel [4]. In that research, roll stability in high speed was the main concern, and two models were tested with several spray rails. It was shown that loss of roll stability in high speed might result in directional instabilities or broaching.

Experimental techniques have been studied in 
vessel maneuvering tests. Several parameters affect the performances of a high-speed vessel, including deadrise angle, longitudinal center of gravity, vertical center of gravity, and trim control fins, which have been investigated in [5].

In order to study the behavior of planing vessel in calm water, restrained hydrodynamic model tests were performed on two models of planing catamarans [6]. These tests included three measuring indices of forces and moments as a function of pitch, heave, roll, drift, and model speed. Furthermore, added mass and damping force of a planing vessel, rudder, and propeller forces were measured in model tests.

In order to have a better understanding of forces and moments acting on a planing vessel while maneuvering in the horizontal plane, the oscillating motion of the high-speed vessel has been investigated in [7]. In this study, the model has been completely restrained and is just under maneuvering forces (pure sway, pure yaw, and yaw with drift). The vessel draft, trim angle, model forward speed, sway speed, and yaw speed change systematically, and forces and moments are measured along each degree of freedom.

In the planing craft maneuver, the running attitude changes, which usually consists of draft, trim, and heel angles [8]. Therefore, prediction of maneuver for the planing craft is more complicated than for other vessels and to accurately simulate maneuverability of these crafts, the motion characteristics (trim angle, heel angle, etc.) are needed [8].

The influences of three longitudinal configurations of demi-hulls on maneuverability of a trimaran have been reported in [9]. In this research, in order to simulate the maneuverability of trimaran vessel, the NUMELS software has been used and it is shown that longitudinal positions of the body sides have a significant influence on the maneuverability of trimarans [10].

Hydrodynamic coefficients of maneuvering have been derived in [11] using the system identification method based on the experimental, analytical tests, and computational fluid dynamic data. They investigated the accuracy of each method in the calculation of the maneuver hydrodynamic coefficients.

In [12], maneuverability of the vessel has been investigated by computational fluid dynamic, before the design phase, and studding maneuvering parameters, such as turning radius and zigzag tests.

In the present paper, a comprehensive model of the planing craft is developed to study the influences of the rudder on the maneuverability of these crafts. Then, straight-line stability, course keeping, and turning circle maneuvers of the planing craft are simulated. To check the accuracy of the method, results of the present numerical method are compared with those of the experiments performed with the model of the planing craft in [7]. It is shown that there is close agreement between the results. Finally, the effects of aspect ratio and the angle of the rudder have been investigated on the maneuverability of planing crafts. As a novel result, it is proven that by using a low-cost and simple method, maneuverability of a planing craft can be predicted accurately and thoroughly.

\section{Planing craft maneuvering equations}

In the analysis of maneuvering and seakeeping of displacement vessels, it is assumed that the wetted surface is constant. However, in planing craft, the wetted surface, draft, and trim angle change rapidly as the forward speed increases [13]. Therefore:

1. All the hydrodynamic coefficients are strongly dependent on the speed;

2. Longitudinal and lateral motions are coupled.

Equations for analyzing the motions of a high-speed vessel are derived according to [13]:

$$
\begin{aligned}
X= & m(\dot{u}+w q-v r) \\
Y= & m(\dot{v}+u r-w p), \\
Z= & m(\dot{w}+v p-u q), \\
K= & \frac{d}{d t}\left(I_{x x} \omega_{x}-I_{x y} \omega_{y}-I_{x z} \omega_{z}\right) \\
& -r\left(I_{y y} \omega_{y}-I_{y z} \omega_{z}-I_{x y} \omega_{x}\right) \\
& +q\left(I_{z z} \omega_{z}-I_{x z} \omega_{x}-I_{y z} \omega_{y}\right), \\
M= & \frac{d}{d t}\left(I_{y y} \omega_{y}-I_{y z} \omega_{z}-I_{x y} \omega_{x}\right) \\
& -p\left(I_{z z} \omega_{z}-I_{x z} \omega_{x}-I_{y z} \omega_{y}\right) \\
& +r\left(I_{x x} \omega_{x}-I_{x y} \omega_{y}-I_{x z} \omega_{z}\right), \\
N= & \frac{d}{d t}\left(I_{z z} \omega_{z}-I_{x z} \omega_{x}-I_{y z} \omega_{y}\right) \\
& -q\left(I_{x x} \omega_{x}-I_{x y} \omega_{y}-I_{x z} \omega_{z}\right) \\
& +p\left(I_{y y} \omega_{y}-I_{y z} \omega_{z}-I_{x y} \omega_{x}\right),
\end{aligned}
$$

where, $\omega$ is the angular velocity of the vessel with respect to the axis and $\Omega=(p, q, r)$ is the angular velocity of the axes.

The force along, the sway direction, and moments in roll and yaw motions are shown in Eq. (2):

$$
\begin{aligned}
& Y=Y_{\dot{v}} \dot{v}+Y_{v} v+Y_{\ddot{\phi}} \ddot{\phi}+Y_{\dot{\phi}} \dot{\phi}+Y_{\phi} \phi+Y_{\ddot{\psi}} \ddot{\psi}+Y_{\dot{\psi}} \dot{\psi}, \\
& K=K_{\dot{v}} \dot{v}+K_{v} v+K_{\ddot{\phi}} \ddot{\phi}+K_{\dot{\phi}} \dot{\phi}+K_{\phi} \phi+K_{\ddot{\psi}} \ddot{\psi}+K_{\dot{\psi}} \dot{\psi}, \\
& N=N_{\dot{v}} \dot{v}+N_{v} v+N_{\ddot{\phi}} \ddot{\phi}+N_{\dot{\phi}} \dot{\phi}+N_{\phi} \phi+N_{\ddot{\psi}} \ddot{\psi}+N_{\dot{\psi}} \dot{\psi} .
\end{aligned}
$$


Table 1. Relations concerning the hydrodynamic coefficients of a planing hull [13].

\begin{tabular}{cc}
\hline Coefficient & Formula \\
\hline$Y_{\dot{V}}$ & $-B^{2} \rho \tan (\beta) k(\beta)\left[L_{k}+2 L_{c}\right] / 12$ \\
$K_{\dot{V}}$ & 0 \\
$N_{\dot{V}}$ & $-B^{2} \rho \tan (\beta) k(\beta)\left[L_{k}^{2}+2 L_{k} L_{c}+3 L_{c}^{2}\right] / 48$ \\
$Y_{\ddot{\psi}}$ & $N_{\dot{V}}$ \\
$K_{\ddot{\psi}}$ & 0 \\
$N_{\ddot{\psi}}$ & $-B^{2} \rho \tan (\beta) k(\beta)\left[L_{k}^{3}+2 L_{k}^{2} L_{c}+3 L_{c}^{2} L_{k}+4 L_{c}^{3}\right] / 120$ \\
$Y_{\ddot{\phi}}$ & 0 \\
$K_{\ddot{\phi}}$ & $0.010237 \rho B^{5} \lambda(1-\sin \beta)+h_{1} Y_{\dot{v}}$ \\
$N_{\ddot{\phi}}$ & 0 \\
$Y_{v}$ & $-0.5 \rho U B^{2}\left[0.6494 \beta^{0.6} T^{2} C_{v}^{2}\right]$ \\
$K_{v}$ & $Y_{v}\left[-K G+1.5145 B / \beta^{0.342}\right]$ \\
$N_{v}$ & $Y_{v}\left[-\mathrm{LCG}+12.384 B T^{0.45} /(\tau+5.28)\right]$ \\
\hline
\end{tabular}

In order to find the coefficients used in Eq. (2), a series of experiments on the hull forms with the deadrise angles of 10, 20, and 30 degrees have been carried out in [13]. In the test procedure, the advance velocity, drift angle, and roll and trim angles are altered and, finally, all the coefficients are defined. The equation concerning each parameter is shown in Table 1.

\section{Rudder forces}

Dimensions and exact location of rudder are not yet determined in the initial design stages; however, only a rough approximation of rudder dimension and position would be enough to evaluate the vessel maneuverability. In the present study, formulations of the rudder forces are based on the studies performed by Inoue and Hooft [6]. They have developed a mathematical model for predicting a ship maneuvering in calm water. Their compact mathematical model includes the hydrodynamic effects caused by seakeeping and maneuvering. They present an accurate theoretical and empirical formula based on the experiments. They assume that the velocities around the rudder are high and flow separation does not occur. By this assumption, the rudder forces formulation is extracted.

In fact, the flow might separate and the present assumptions might somehow affect the accuracy. Furthermore, in the present study, the added mass and damping of the rudder are completely ignored. The local flow velocity entering into rudder can be calculated using Eqs. (3) to (5):

$$
\begin{aligned}
& u_{r}=U\left(1-w_{p}\right)+C_{D U} \cdot \Delta u_{p}, \\
& v_{r}=C_{d b}\left(v \cos \phi_{F}+w \sin \phi_{F}\right)-C_{d r} \sqrt{x_{r}^{2}+y_{r}^{2}} \cdot r \\
& +C_{d r} \sqrt{x_{r}^{2}+z_{r}^{2}} \cdot q \\
& U_{\text {rud }}=\sqrt{u_{r}^{2}+v_{r}^{2}} \text {. }
\end{aligned}
$$

In Eq. (3), the effects of velocity increment are calculated using Eq. (6):

$$
C_{D U}=0.7 \frac{D_{P}}{h_{r}}, \quad \text { or } \quad 0.9 \frac{D_{P}}{h_{r}} .
$$

In Eq. (4), $\phi_{F}$ is the relative angle between the rudder and vertical plane. $z_{r}, y_{r}$, and $x_{r}$ represent position of the rudder relative to the vessel's center of gravity. The effective angle of incidence of the flow to the rudder is:

$$
\delta_{e}=\delta-\delta_{H}
$$

The lateral rudder force can be determined as [6]:

$$
L=\frac{1}{2} \rho A_{t} C_{L \delta} U_{\mathrm{rad}}^{2} \sin \delta_{e}
$$

where:

$$
C_{L \delta}=\frac{6.13 A_{e}}{A_{e}+2.25} .
$$

The lift-induced drag in the direction of the rudder inflow is described by:

$$
D=\frac{1}{2} \rho A_{t} C_{D i} U_{\mathrm{rad}}^{2} \sin \delta_{e}
$$

in which:

$$
C_{D i}=\frac{C_{L \delta}^{2}}{\pi A_{e}}
$$

The friction resistance of the rudder due to its friction drag is formulated as:

$$
R=\frac{1}{2} \rho S_{w T} C_{T R}\left(U_{\text {rad }}^{2} \sin \delta_{e}\right)^{2} .
$$

The normal force on the rudder due to the lateral drag coefficient, $C_{n}=1.8$, is:

$$
Y_{N}=\frac{1}{2} \rho A_{r} C_{N} U_{\mathrm{rad}} \sin \delta_{e}\left|U_{\mathrm{rad}} \sin \delta_{e}\right|
$$


Therefore, following equations will define the forces and moments exerted on the rudder:

$$
\begin{aligned}
X_{\text {rud }}= & -R \cos \delta-D \cos \delta_{H}-Y_{N} \sin \delta-L \sin \delta_{H}, \\
Y_{\text {rud }}= & \left(-R \sin \delta-D \sin \delta_{H}+Y_{N} \cos \delta\right. \\
& \left.+\left(1+a_{h}\right) L \cos \delta_{H}\right) \cos \phi_{F}, \\
Z_{\text {rud }}= & \left(-R \sin \delta-D \sin \delta_{H}+Y_{N} \cos \delta\right. \\
& \left.+\left(1+a_{h}\right) L \cos \delta_{H}\right) \sin \phi_{F}, \\
M_{\text {rud }}= & X_{\text {rud }} \cdot z_{r}+Z_{\text {rud }} \cdot x_{r}, \\
N_{\text {rud }}= & -X_{\text {rud }} \cdot y_{r}+\left(\left(Y_{N} \cos \delta-R \sin \delta-D \sin \delta_{H}\right) \cdot x_{r}\right. \\
& \left.+\left(x_{r}+a_{h} \cdot x_{h}\right) L \cos \delta_{H}\right) \cos \phi_{F} .
\end{aligned}
$$

\section{Model Specifications}

In order to check validity of the present numerical method in simulating the maneuver of the planing vessel, a model-scaled planing vessel is considered, for which experimental data on its maneuvering is available in publications. The characteristics of the boat model used in the simulation were taken from [7]. The characteristics and the body plan of the model are presented in Table 2 and Figure 1.

In the following sections, different types of maneuvers will be studied and the behavior of the vessel will be analyzed.

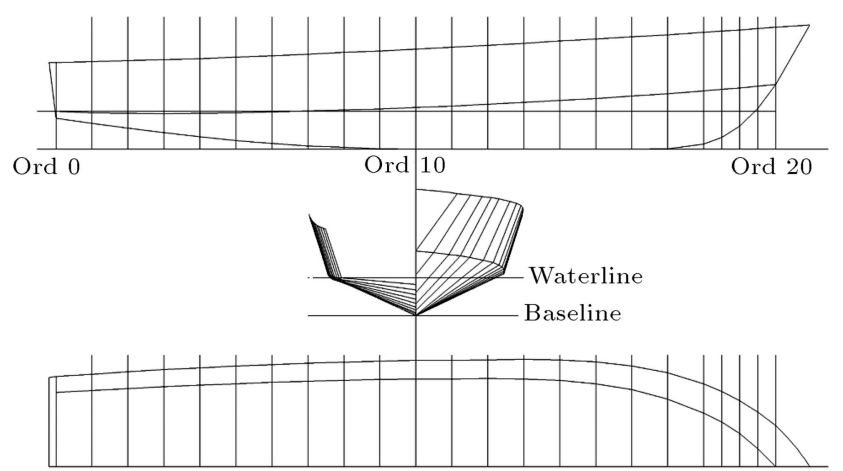

Figure 1. Body plan of the model [7].

Table 2. Characteristics of the model.

\begin{tabular}{clc}
\hline Parameter & \multicolumn{1}{c}{ Description } & Value \\
\hline$L$ & Length $(\mathrm{m})$ & 1.5 \\
$B$ & Breadth $(\mathrm{m})$ & 0.367 \\
$M$ & Mass $(\mathrm{kg})$ & 11.5 \\
$\tau$ & Trim (degree) & 5 \\
$\beta$ & Deadrise (degree) & 10 \\
$L_{c g}$ & Longitudinal center of gravity & 0.726 \\
& measured from stern $(\mathrm{m})$ & \\
\hline
\end{tabular}

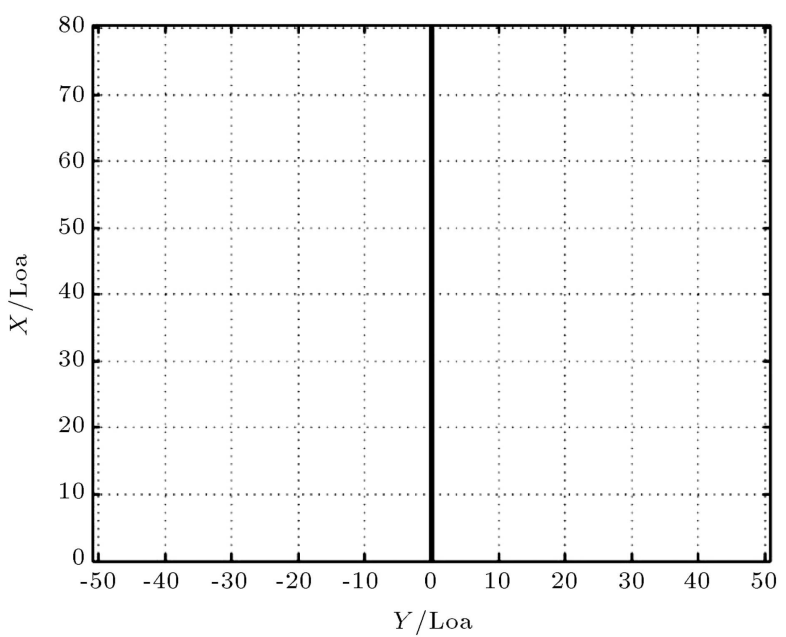

Figure 2. Vessel path with zero rudder angle.

\section{Simulation of motion along the straight line}

A computer program is developed using Matlab programmer to simulate the maneuvering of a planing craft. Inputs of this program are characteristics of the model shown in Table 2, i.e. rudder angle and forward speed of the model. Outputs of the program are ship speeds in surge, sway, yaw directions, its positions, and yaw angle in each time.

For simulation of the maneuvering along the straight line, the rudder angle is set to zero and forward speed is set to $5 \mathrm{~m} / \mathrm{s}$ in the range of the planing craft.

The vessel path of the boat with zero rudder angle is shown in Figure 2. As it is expected, the path is a straight line and no lateral speed has been developed. Therefore, the numerical prediction is in total agreement with what is expected from a real ship.

\section{Simulation of course-changing maneuver}

The aim of the course-changing maneuver in a vessel is to find out sensitivity of the vessel's motion to the rudder angle. In order to perform this maneuver, the rudder angle is set to a specific constant angle, while the vessel moves ahead in a straight line. The vessel starts to change its path from the straight line. When the angle between new directions of the vessel and its initial straight-line path reaches the value of the set rudder angle, this test is ended and the corresponding time duration is logged. According to the logged time duration and with the help of the longitudinal motiontime diagram of the vessel, the traveling distance of the vessel can be calculated. In order to perform this maneuver test in the present study, the initial vessel speed and its ruder angle are set to $5 \mathrm{~m} / \mathrm{s}$ and 15 degrees, respectively.

The numerical software was run with the mentioned initial conditions. The results for traveling path 
of the vessel, vessel yaw diagram versus time, and the longitudinal path traveled by the vessel versus time are shown in Figures 3 to 5, respectively. In the yaw diagram of the vessel, the corresponding time that the yaw angle of the vessel took to reach 15 degrees was logged. Then, using the longitudinal displacement diagram of the vessel along the surge motion, its traveled distance was determined. This procedure was repeated for the negative angle of the rudder, which had the same result.

As seen in Figure 4, when the rudder angle is set to 15 degrees, it takes 2.7 seconds for yaw angle of the vessel to reach 15 degrees. In this time duration, the vessel travels about 15 times its own length according to Figure 5. The displacement along the surge direction looks linear, but it is not true linear. Its rate increment decreases as the vessel approaches 90 degrees. This traveled path can be compared to those of the design guidelines, if any exists.

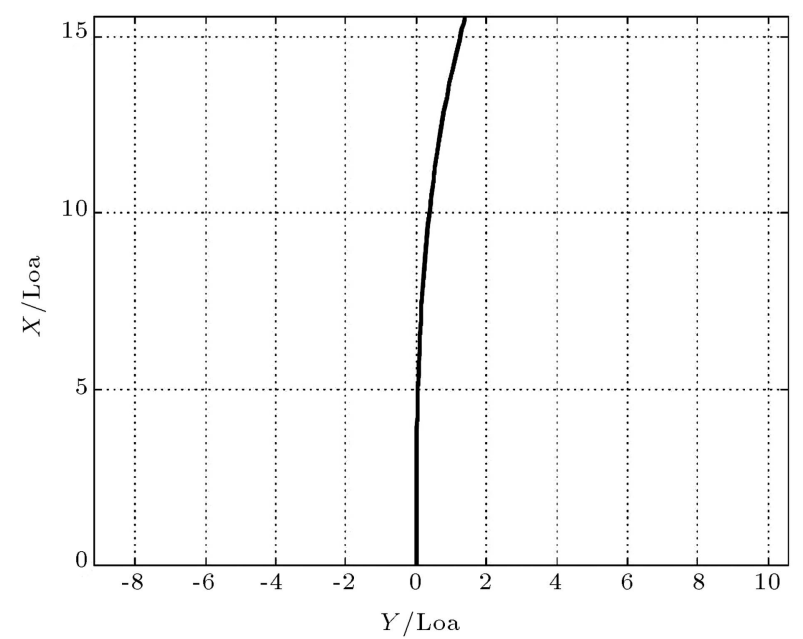

Figure 3. Vessel traveling path corresponding to the rudder angle of 15 degrees.

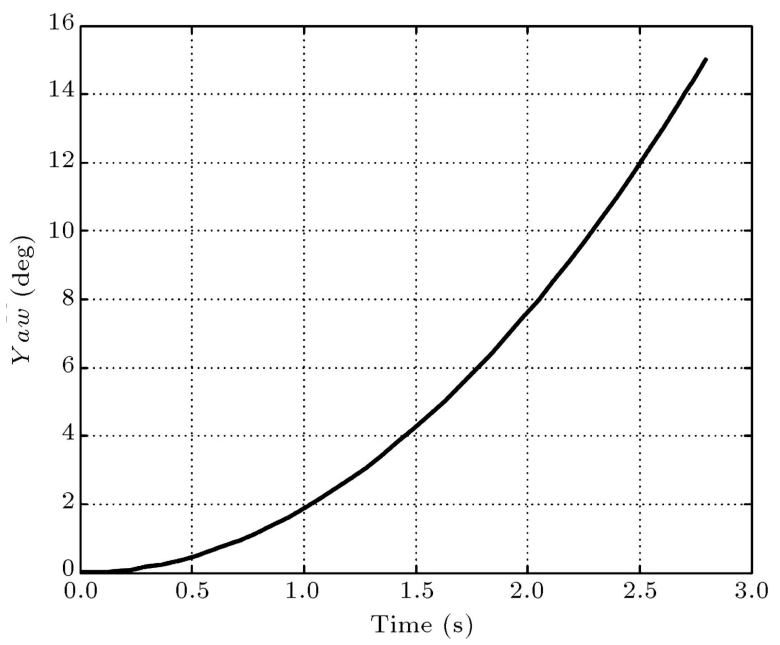

Figure 4. Vessel yaw angle corresponding to the rudder angle of 15 degrees.

\section{Simulation of the turning circle maneuver}

In some situations (like collision avoidance), it is necessary for a vessel to turn while keeping stability in the maneuvering. According to Figure 6, in the turning circle maneuver, at first, the vessel travels in a straight line with a constant speed and, then, the rudder angle is set to its maximum and kept fixed until the vessel travels a whole circle (at least 540 degrees). This test should be performed for both portside and starboard sides of the vessel.

For simulation of the turning test, it was consid-

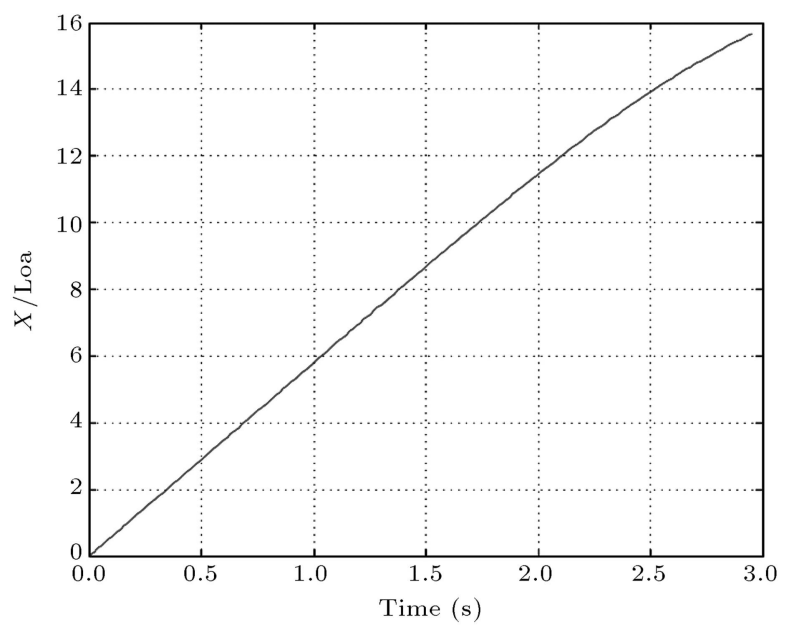

Figure 5. Vessel displacement along the surge motion with the rudder angle of 15 degrees.

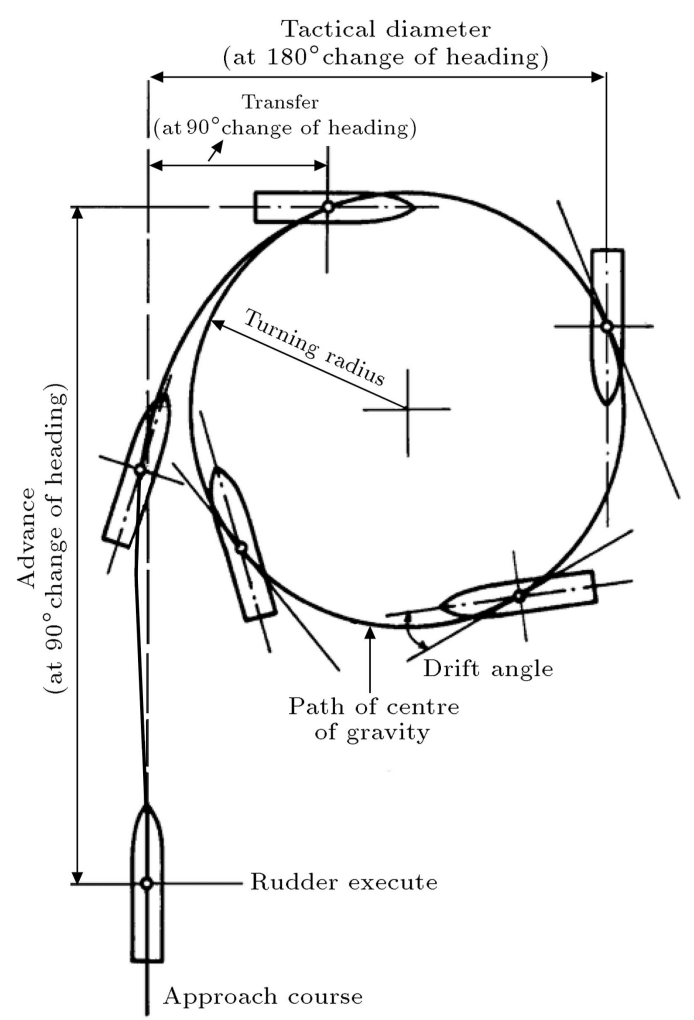

Figure 6. Definitions used in turning test [14]. 


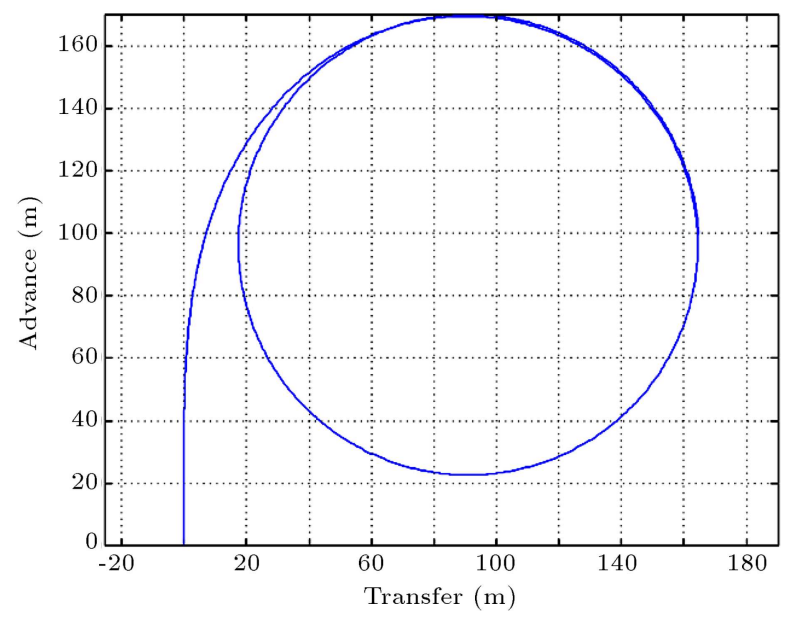

Figure 7. Circling maneuver simulation.

ered that the vessel was moving ahead with a speed of $10.3 \mathrm{~m} / \mathrm{s}$. Also, it was assumed that the vessel had reached the stable condition and no environmental forces were affecting it. Then, the rudder angle was set to 19 degrees and the simulation was carried out. The simulated turning circle of the vessel is shown in Figure 7.

In the diagram of Figure 7, it can be found that:

- The vessel advancement is $170 \mathrm{~m}$;

- The tactical diameter of the turning circle is $150 \mathrm{~m}$;

- The vessel transfer is about $90 \mathrm{~m}$.

In [7], the turning circle maneuver of the present model with the same scenario used in the present paper was experimentally performed. Results of the present study and those of [7] are shown in Figure 8.

As seen in Figure 8, there are some differences in the results, for which the source of the errors can

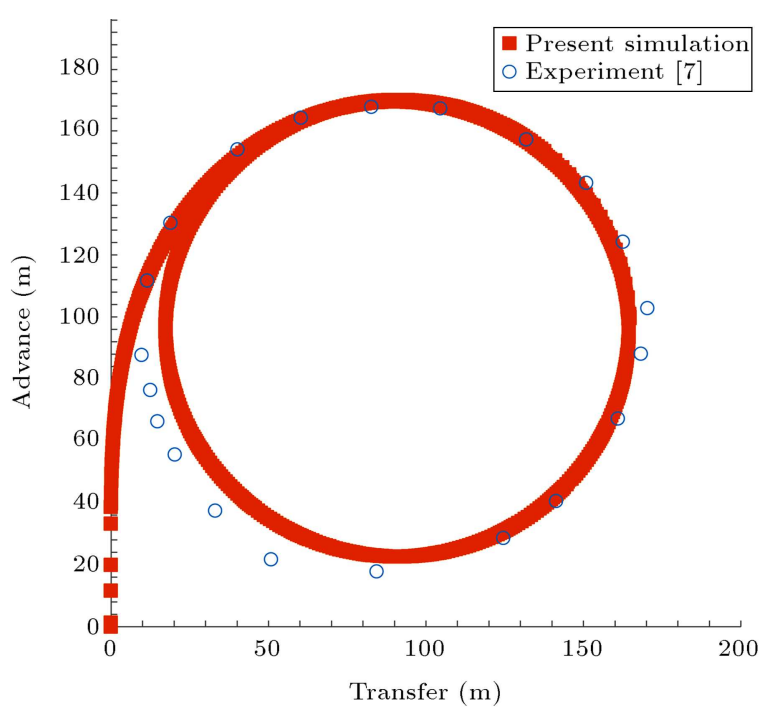

Figure 8. Turning circle maneuver of the present method and results in [7]. be discussed. The hydrodynamic coefficients used in numerical modeling of the turning maneuver are taken from Table 1; these coefficients are applicable only for the planing craft with prismatic hulls. However, the hull form used in the experiments is not prismatic; therefore, existence of such errors is justified.

\section{Rudder influence on maneuverability of the planing hull}

Forces exerted on a vessel while maneuvering in calm water are from different sources. From this viewpoint, forces can be divided into two distinct categories. First, as the vessel maneuvers, the angle of attack to the incident flow leads to hull hydrodynamic forces as described previously. The other category is external forces due to control surfaces such as the rudder. In the case of displacement vessels, the rudder forces are small when compared to huge hull forces. But, in the case of planing vessels, the rudder forces are compared to the main hull forces. Rudder forces are proportional to square of the inlet velocity. Because of the high inlet flow velocity to rudder, the forces generated by a lifting surface, such as the rudder, are extremely high. Thus, the rudder is much more effective in the case of high-speed crafts.

Some geometric characteristics of rudder govern the produced forces. As it was mentioned, the flow velocity is the main concern. Angle of attack (rudder angle) and aspect ratio are prominent too according to Eqs. (8) and (9).

In the following, the effect of rudder angle on turning circle of the planing craft is investigated. The equations of motions are solved for three rudder angles of 17, 19, and 21 degrees. Simulated turning circle maneuvers are shown in Figure 9.

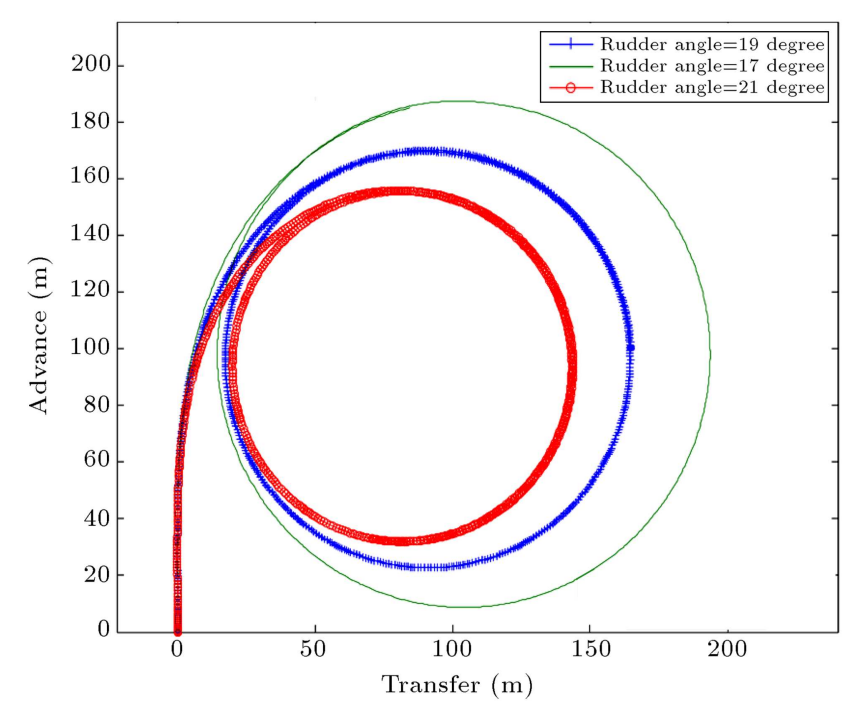

Figure 9. Influence of the rudder angle on turning circle maneuver. 


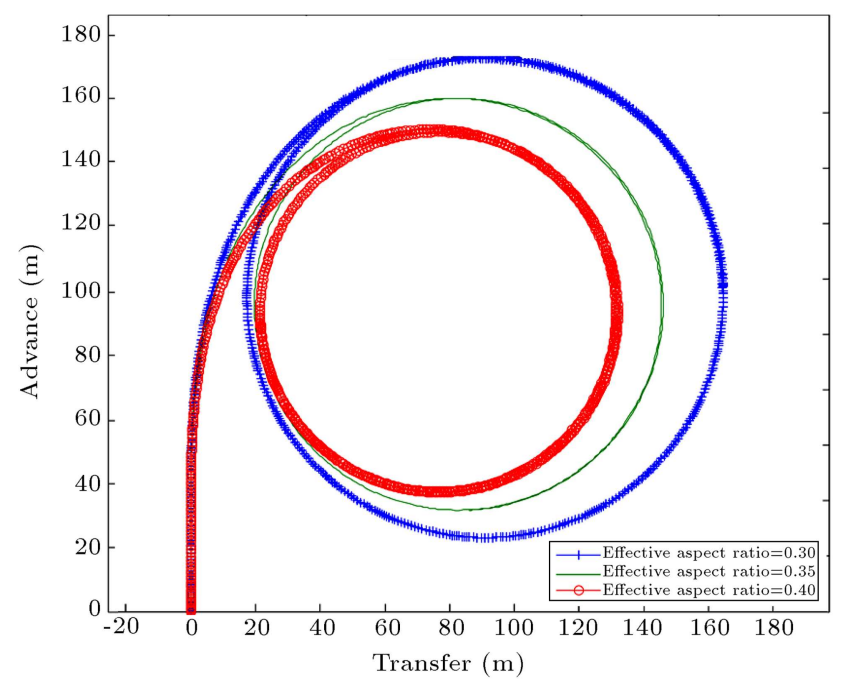

Figure 10. Turning circle maneuvers for different aspect ratios.

As seen in this figure, the tactical diameter slightly decreases, from 170 to 100 meters, as the rudder angle increases from 17 degrees to 21 degrees. As the rudder angle increases, the generated lift force increases too. On the other hand, the rudder drag would also be high. Thus, the speed loss due to turning would be greater for high rudder angles.

Rudder geometry and dimensioning are the main concerns of designers in the early stages of high-speed craft design cycle. One of the main characteristics is the rudder aspect ratio. Rudder lift/drag efficiency for a given angle of attack is strongly influenced by the aspect ratio, which is the depth or span of the rudder divided by the average chord length as shown in Eq. (15). Larger aspect ratios, with the same area, result in more efficient rudders and generate a given amount of lift at low angles of attack, in comparison with rudders with lower aspect ratios [15]:

$$
A R=\frac{T}{C}
$$

The simulation is repeated for three rudders with different aspect ratios and the results are presented in Figure 10. It can be deduced that the aspect ratio is an important design parameter. Greater aspect ratio leads to less tactical diameter of the vessel; with increasing the aspect ratio from 0.3 to 0.4 , the tactical diameter decreases from 150 to 100 meters. Aspect ratio is a control parameter in the design stage and ship maneuverability can be modified by optimization of rudder aspect ratio.

\section{Conclusion}

In the present study, a mathematical model was presented, in which the influences of the roll angle,

pitch angle, and vertical displacement of the ship were considered. The proposed model was verified by using experimental results of an available planing model. This model accurately predicts high-speed maneuvering of the vessel and the results are in a good agreement with those of [7]. The vertical position of the center of gravity, weight distribution, and the deadrise angle are inputs of the developed computer programmer to simulate maneuvering of the planing crafts. Furthermore, a simple mathematical model, which can be used for planing vessels and controlling their traveling path, is an important issue, which has been formulized in this paper. Rudder selection is a main concern in the design of high-speed crafts and, hence, the effect of rudder on vessel maneuverability has been studied in this paper. The mathematical model was used for comparison of different rudder parameters of the turning circle. It was shown that by increasing the amount of aspect ratio of the rudder to $50 \%$, the turning circle diameter was reduced to $25 \%$.

\section{Nomenclature}

Surge linear velocity

Sway linear velocity

Heave linear velocity

Angular velocity of roll motion

Angular velocity of pitch motion

Angular velocity of yaw motion

Forces exerted on the vessel along the surge motion

Force exerted on the vessel along the sway motion

Force along the heave motion

Moment of roll motion

Moment of pitch motion

Moment of the yaw motion

Average wetted chine beam

Deadrise angle amidships

Dynamic trim (positive bow up)

Wetted length of chine

Wetted length of keel

Transom draft

Speed coefficient $(U / \sqrt{g B})$

Longitudinal Center of Gravity (measured from transom)

Mean wetted length/beam ratio, $\left[L_{K}+L_{C}\right] / 2 B$

Hull added mass function: $0.06641+$ $0.00716 \beta+0.0003861 \beta^{2}, \beta(\mathrm{deg})$ 
$K G \quad$ Height of the CG from the keel

I Moment inertia

$m \quad$ Ship mass

$\delta_{e} \quad$ Effective angle of incidence of the flow to the rudder

$\delta \quad$ Rudder angle

$L \quad$ Lateral rudder forces

$h_{r} \quad$ Rudder height

$c_{r} \quad$ Rudder cord length

$A_{r} \quad$ Rudder lateral surface

$A_{e} \quad$ Effective aspect ratio of rudder

\section{References}

1. Ye. Li, "The simulation of ship maneuvering \& course keeping with escort tus", M.S. Thesis, Department of mechanical engineering, University of British Columbia, pp. 13-60 (2004).

2. ITTC Recommended Procedures \& Guidelines, Testing and Extrapolation Methods Manoeuvrability Free Running Model Tests, pp. 1-10 (2005).

3. Fossen, T.I. "A nonlinear unified state-space model for ship maneuvering \& control in a seaway", Journal of Bifurcation and Chaos, pp. 1-28 (2005).

4. Suhrbier, K.R. "An experimental investigation on the roll stability of a semi-displacement craft at forward speed", Symposium on Small Fast Warship and Security Vessels, The Royal Institution of Naval Architects, Paper No. 9, pp. 133-142 (1978).

5. Deakin, B. "Model tests to assess the manoeuvring of planing craft", The International HISWA Symposium on Yacht Design and Yacht Construction, pp. 21-32 (2005).

6. Toxopeus, S.L. Keuning, J.A. and Hooft, J.P. "Dynamic stability of planing ships", International Symposium and Seminar on the Safety of High Speed Craft, London, UK, pp. 1-16 (1997).

7. Plante, M., Toxopeus, S., Blok, J. and Keuning, A. "Hydrodynamic maneuvering aspects of planing craft", International Symposium and Workshop on Forces Acting on a Manoeuvring Vessel, Val de Reuil, FranceV, pp. 1-9 (1998).

8. Katayama, T., Taniguchi, T., Fujii, H. and Ikeda, Y. "Development of maneuvering simulation method for high speed craft using hydrodynamic forces obtained from model tests", 10th International Conference on Fast Sea Transportation FAST 2009, Athens, Greece, pp. 477-489 (2009).

9. Javanmardi, M.R., Seif, M.S., Jahanbakhsh, E. and Sayyaadi, H. "Trimaran maneuvering simulation, based on a three-dimensional viscous free surface flow solver", Proceeding of 6th International Conference on High-Performance Marine Vehicles (HIPER'08), Naples, pp. 249-256 (2008).

10. Jahanbakhsh, E., Panahi, R. and Seif, M.S. "Catamaran motion simulation based on moving grid technique", Journal of Marine Science and Technology, 17(2), pp. 128-136 (2009).

11. Araki, M., Sadat-Hosseini, H., Sanda, Y., Tanimoto, K. and Umeda, N. "Estimating maneuvering coefficients using system identification methods with experimental", System-Based, and CFD Free-running Trial Data, Ocean Engineering Journal, 51, pp. 63-84 (2012).

12. Simonsen, C.D., Otzen, J.F., Klimt, C. and Larsen, N.L. "Maneuvering predictions in the early design phase using CFD generated PMM data", 29th Symposium on Naval Hydrodynamics, Gothenburg, Sweden, pp. 26-31 (2012).

13. Lewandowski, E.M. "The dynamics of marine craft: maneuvering and seakeeping", Advanced Series on Ocean Engineering, 22, Copyright 2004 by World Scientific Publishing Co. Pte. Ltd, pp. 50-120 (2004).

14. Bertram, V., Practical Ship Hydrodynamics, British Library Cataloguing in Publication Data, First published, pp. 150-200 (2000)

15. Patton, M.R. "Evaluation of dynamic lift coefficients of high aspect ratio rudders and control surfaces", United States Naval Academy Annapolis, Maryland, U.S.N.A. Trident Scholar project report; pp. 1-134 (2004).

\section{Biographies}

Sajad Hajizadeh received the BSc degree in Naval Architecture from Persian Gulf University, Busher, Iran, in 2007 and the MSc degree in Marine Engineering from Sharif University of Technology, Tehran, Iran, in 2010. He is currently PhD student in the School of Mechanical Engineering at Sharif University of Technology, Tehran, Iran. His main areas of interest are hydrodynamic and maneuvering of marine vehicles. He has published several papers in this field.

Mohammad Saeed Seif received the BSc degree in Mechanical Engineering from Amirkabir University of Technology, Tehran, Iran, in 1989; the MSc degree in Naval Architecture from Technical University of Gdansk, Gdansk, Poland, in 1992; and PhD degree in Mechanical Engineering from Yokohama National University, Yokohama, Japan, in 1995. He is currently Professor in the Department of Mechanical Engineering, Sharif University of Technology, Tehran, Iran. His area of expertise is dynamics and hydrodynamics of marine vehicles, ship structure design, advanced marine vehicles, and advanced fluid dynamics. 
He has published several papers in the maneuvering field.

Hamid Mehdigholi received the BSc degree in Mechanical Engineering from Sharif University of Technology, Tehran, Iran, in 1977 and the MS and PhD degrees in Applied Mechanics from Imperial College, London, in 1986 and 1991, respectively. He has been a Faculty Member at Sharif University of Technology since 1991. His research interests include dynamics of rotating machines, vibration isolation, acoustics, and experimental modal analysis. 